\title{
Self-organising map methods in integrated modelling of environmental and economic systems
}

\author{
$\underline{\text { S Shanmuganathan }}^{\mathrm{a}}$, P Sallis $^{\mathrm{a}}$ and J Buckeridge ${ }^{\mathrm{b}}$ \\ ${ }^{\mathrm{b}}$ Environmental and Oceanic Sciences Centre, \\ a Auckland University of Technology, New Zealand. subana.shanmuganathan@aut.ac.nz
}

\begin{abstract}
The need for better techniques, tools and practices to analyse ecological and economic systems in an integrated framework has never been so great. Self-organising map ${ }^{1}$ (SOM) methods are utilised for this purpose with two examples using regional and global data (ecological and economic) compiled by state and international institutions i.e. Waikato Regional Council and the World Bank. Sustainable ecosystem management through holistic or interdisciplinary approaches such the triple bottom line, 4Es (economics, ecology, ethics and engineering) concepts has been emphasised for a long time now. Many national and international institutions have been investigating for integrated, forward looking management practices i.e. integrated assessment management (IAM) by scientists and the pressure, state and response (PSR) model by the Organisation for Economic Cooperation and Development (OECD), World Resources Institute, the World Bank and Ministry for the Environment, New Zealand. Despite these efforts our understanding of ecosystem response to human influence is insufficient to carry out proper impact assessment on proposed developmental activities. Thus, in practice the implementation of sustainable environmental management seems remote. While the environmentalists and developers wrangle over the reliability of current environmental impact assessment practices and their results ecosystems continue to deteriorate, with commensurate biodiversity loss. The examples of this paper utilising SOMs to analyse disparate data sets at these different scales produce potential for future use: (i) regional, from river water quality monitoring to evaluate ecosystem response to human influence and (ii) global, for modelling environmental and economic data and trade-off analysis within an integrated framework to inform sustainable environmental management.
\end{abstract}

Key words: Integrated modelling techniques; Ecological modelling; Self-organising maps; Ecological data

\section{INTRODUCTION}

The need for better techniques, tools and practices to analyse ecological and economic systems in an integrated framework has never been so great. Many concerned over the global environmental issues i.e. scientists and international institutions do agree that in spite of the progress achieved so far, better techniques and tools are needed to model the naturally evolving, highly complex and diverse ecosystems so that the effects of human influence on them are sustained in the long run. Even though many recent articles have discussed the issues, recommending innovative measures for integrated analysis of ecological and economic data, none of them has been practical. Stakeholders and land developers not concerned over the issues, continue to ignore the disturbing scientific predictions and argue upon the reliability of current environmental impact assessment methods. Meanwhile ecosystems continue to deteriorate at global scales with commensurate biodiversity loss. Thus, in practice achieving sustainable environmental management seems remote. The urgent need for new approaches and the drawbacks with current methods of ecological modelling are explained.
Self-organising $\operatorname{map}^{1}$ (SOM) approaches for integrated analysis of ecological and economic data (at local and global scales) are then illustrated.

\section{NEED FOR INTEGRATED MODELLING}

In view of the global environmental issues, integrated assessment and modelling (IAM) could provide a means to bring scientists, policymakers and the societies involved, together in order to solve the issues, Harris (2002). Scientific predictions based on highly complicated principles and hypotheses, are generally beyond comprehension by individuals of different professions thus seen ignored by the latter. This has been a common practice for a long time. Scientists are getting more and more focused on science and research becoming poor communicators, Buckeridge (2001), and isolated

\footnotetext{
1 Self-organising maps (SOMs) belong to a paradigm of feed forward artificial neural networks (ANNs) that are based on unsupervised algorithmic processing. They are capable of projecting multidimensional input data onto low dimensional displays, enhancing the detection of useful knowledge from the data impossible by traditional methods.
} 
from the rest, especially with the general public. The knowledge divide is seen to be the main reason for the ignorance of useful scientific predictions by stakeholders and the general public Clark et al. (2001).

New modelling techniques, radical approaches and better rapport are described to be of paramount importance for establishing better communication between the three main groups. Vant (1999); Reid (2000); Clark et al. (2001); Harris (2002) all stressed that better modelling tools with an integrated approach could play a significant role in enhancing a common trust between these different groups. The use of tools to depict disparate data (i.e. from different disciplines) is described to be vital in the efficient use of natural systems without any biodiversity loss. New tools are envisaged to improve our understanding and provide a means for prediction of complex ecosystems in response to a variety of human influence and other natural causes.

\subsection{Ecological Forecasts: An Emerging Imperative}

Continuous forecasts on ecosystem behaviour with suggestions for trade-offs, alternative options and evaluation of feedbacks are vital for sustainable environmental management. The current environmental problems such as rapid change in climate and chemical cycles, depletion of the natural resources those support regional economies, proliferation of exotic species, spread of disease and deterioration of air, waters and soils all pose unprecedented threats to human civilisation. Had humans implemented better planning and decision making based on reliable forecast on ecosystem state and functioning, we would not have been witnessing any of the above stated environmental problems, Clark et al. (2001).

The slow ecosystem responses that are invariably left out in the current ecological modelling techniques should be included. The 'large inherent uncertainty' arising from strong nonlinearities and stochasticity could not be explained as the impact on ecosystem being neutralised or mitigated. Instead, the 'slow variables', that could be significant in ecological processes should be identified and used for ecosystem modelling, Clark et al. (2001).

A similar approach could be seen in the early 1980s modelling efforts. For example, Mann (1982) stated that ecosystem modellers should focus on developing theories and models to establish the connection between the dynamics of populations and the behaviour of ecosystems, similar to that of the statistical mechanics, which provides a common connection between the motion of particles and the behaviour of a gas. The 'slow variable' in Clark et al. (2001) could be compared to the 'dynamics of populations' of Mann (1982) as an indicator of ecosystem behaviour. However, the current models and approaches in ecology do not have the ability to investigate something of which our prior knowledge is rather poor; in terms of such slow patterns of responses. A similar situation encountered in industrial system modelling is considered to have successfully overcome with the use of SOMs by making use of the measurable system variables, recorded on-line. Using the approach Shanmuganathan et al. (2001) modelled the Long Bay Okura Maine Reserve monitoring data to unravel the non-linear relationships of the Reserve's ecosystem.

\subsection{Major challenges in monitoring programs: transformation of abundant data into information}

"A major challenge for many environmental monitoring programmes is the transformation of large quantities of data into simple, useable information. There are generally two key parts to this:

(i) identifying robust methods for summarising lots of data, while avoiding oversimplification and

(ii) presenting the resulting information in an understandable and attractive way to a largely non-technical audience ${ }^{1}$.

In many respects the need to simplify and display our information and conclusions represent a major challenge to environmental scientists. Indeed, many of us were probably attracted to the study of ecosystems because of their complexity, and now increasingly find ourselves having to move beyond the security of erudite conceptual models and the jargon or our technical journals to communicate with the wider community." Vant (1999:1).

\subsection{Integrated and forward-looking approach to ecosystem management}

An example from Reid (2000), illustrated the state of a native fish of Lake Victoria in Africa. In the example, the new introduced species were found succeeding beyond expectations with a dramatic growth in fish harvest. One of the non native species, Nile perch was found flourishing

\footnotetext{
"At the same time, the 'traditional' use of monitoring data as the basis for rigorous technical appraisals (e.g., Vant \& Bellingaham 1997) should not be overlooked; the main focus of this paper, however, is on other less-technical uses of data."
} 
and which in the end led to the extinction of 350 native species, or reduced to a fraction of their original size.

Many resource management decisions and actions of the past, solely to meet one need, described by Reid (2000) as "... examples abound of vast and uncontrolled ecosystem 'experiments'...." led to an array of unforeseen side effects. Some of them are even described to be responsible for altering the natural flows and cycles of the Earth. The following are some such global issues based on Reid (2000).:

(i) The expansion of agricultural land into natural habitats around the word increased food production but changed the quantity and quality of freshwater runoff tremendously.

(ii) The use of modern fertilisers, as expected increased the yield but also caused eutrophication of nearby rivers and estuaries, further was found to be responsible for anoxic 'dead zones' seen in coastal areas near major agricultural river basins.

(iii) Timber harvest and transformation of forestland to agriculture helped many states to meet their needs for food and fibre but also released carbon into the atmosphere that changed Earth's surface reflectivity, contributing significantly to the risk of global climate change

The inability to understand the various processes of an ecosystem as a whole has led to the failures in forecasting the side effects of human influenced activities on them. Buckeridge (1994); Reid (2000); Gustavsson (2001) emphasised the need for a systems/ integrated/ holistic approach for modelling ecosystems. Research on ecosystem processes (i.e. environmental and biological) of the twentieth century became very focused looking into details and gaining in-depth knowledge with highly specialised scientific disciplines. It has encouraged a fragmented image of nature and is blamed for significantly contributing to the current environmental problems, Bowler (1992).

\subsection{Grand challenges of this era}

A committee appointed by the National Research Council of the National Science Foundation in the United States to select research initiatives for immediate focus in environmental sciences chose the following aspects among the eight areas of 'highest priority': “...

- Biological Diversity and Ecosystem Functioning: an initiative to develop a comprehensive understanding of the factors that generate, maintain, and diminish biological diversity and their effects on ecosystem functioning.

- Hydraulic Forecasting: an initiative to develop a comprehensive hydrological forecasting, specifically including the ecological consequences of changing water regimes.

- Infectious Disease and the Environment: an initiative to develop a comprehensive ecological and evolutionary understanding of infectious and environmental diseases.

- Land-Use Dynamics: an initiative to develop a systematic, spatially explicit understanding of the changes in land use and land cover that are critical to ecosystem functioning, ecosystem services, and human welfare...." Graedel et al. (2001:60).

"...The overall effort will require interdisciplinary research involving ecologists, ethnologists, psychologists, engineers, economists, planners, landscape architects and others. The definitions of data needs and the collection and synthesis of data will require cooperation among physical, biological, and social scientists; engineers and planners; and other associated funding agencies." Graedel et al. (2001:62).

Coordinated research in the following areas with environmental science was suggested to gain more understanding on the controls and means of protecting biodiversity:

(i) To coordinate research on hydraulic modelling on runoff and subsurface water, which reflect the way, living beings, humans inclusive, interact with the landscape.

(ii) To include effects of human management institutions on ecosystems. Include the effects of changing patterns of land use and land cover on potential for habitat redesign.

(iii) To include the effects of climate change in ecosystem functioning assessments and in habitat design to buffer for disturbances and extreme events.

(iv) To create partnerships and work with urban long-term ecological research sites.

The scientific papers so far discussed, described the urgent need for better modelling techniques in order to better understand the effects of human influence on ecosystems. 


\section{SOMS IN REGIONAL DATA ANALYSIS}

Routine measurements of river water quality in the Waikato region began in 1980 . The aim of this programme was to monitor the conditions of water at several sites of this River (figure 1), the largest and most intensively used in the region. The extent of the datasets from these monitoring sites poses a huge challenge to analyse them. The more recent extension with 100 monitoring sites on the other rivers and streams of the region has further complicated the analysis of the twodecade-old data Vant (1999). The following are the parameters used in the SOM:

(i) \%DO: \% Dissolved Oxygen

(ii) Cond: Conductivity - Lab Meter

(iii) DO: Dissolved Oxygen

(iv) DOC: Dissolved Non Purgeable Org. C

(v) DRP: Dissolved Reactive Phosphorus

(vi) Ecoli: Escherichia Coliforms - MF MFC/NA-MUG

(vii) FC: Faecal Coliforms - MF MFC

(viii) FS: Faecal Streptococci - MF

(ix) HardT: Hardness Total

(x) HCO3: Bicarbonate

(xi) HPC: Heterotrophic Plate Count 35"C

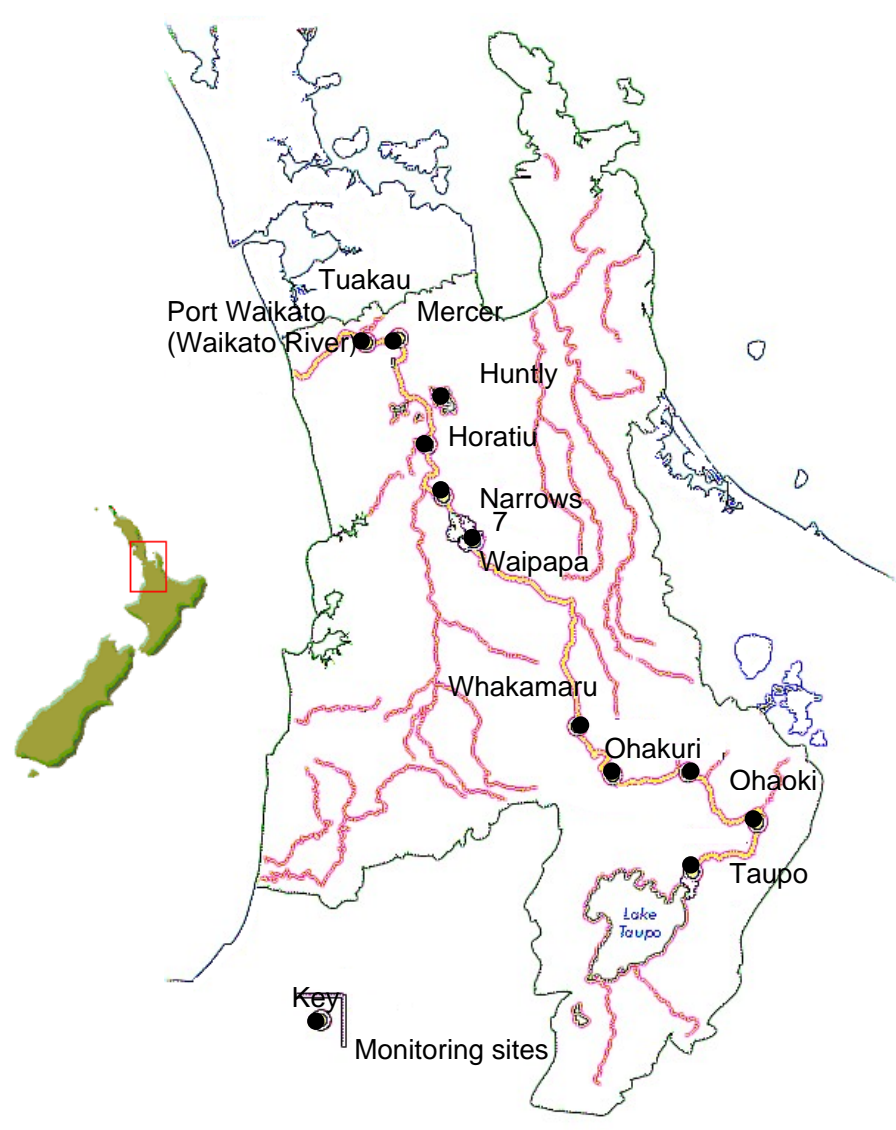

Figure 1: Monitoring sites of the Waikato River. Source: Waikato Regional Council. (xii) NO3: Nitrate Nitrogen

(xiii) SS: Suspended Solids - direct samp TP: Total Phosphorus - Method 4500-PE

(xiv) TPN: Tot Persulphate Nitrogen

(xv) Turb: Turbidity - HACH $2100 \mathrm{~N}$

(xvi) Wtemp: Water Temp - meter

(xvii) ZnD: Zinc Dissolved

(xviii) ZnTR: Zinc Total Recoverable

\subsection{SOM results of the Waikato river data}

The monitoring data, stored in computer databases has been used only in highly technical reports that are found to be of little use to resource managers or the public Vant (1999). In recent times, the role of these two groups is considered to be equally significant as that of scientists in preventing our global ecosystem. The following are the observations made from the SOM (figure 2a):

(i) Taupo control gate data are seen on the left side with Mercer branch on the right and top centre of the SOM; this reveals decrease in DO along with increase in $\mathrm{NO}_{3}$ as human use increases.

(ii) Mercer Branch data seen in the right top centre with little higher water temperature and DO than in the right side of the SOM, are from 1987 onwards; this indicates an increase in water temperature since then.

On the SOM of the river monitoring data (19802002), a trajectory for 4 June 2002 data was run to see the water quality changes, as human use increased (figure $2 b$ ).

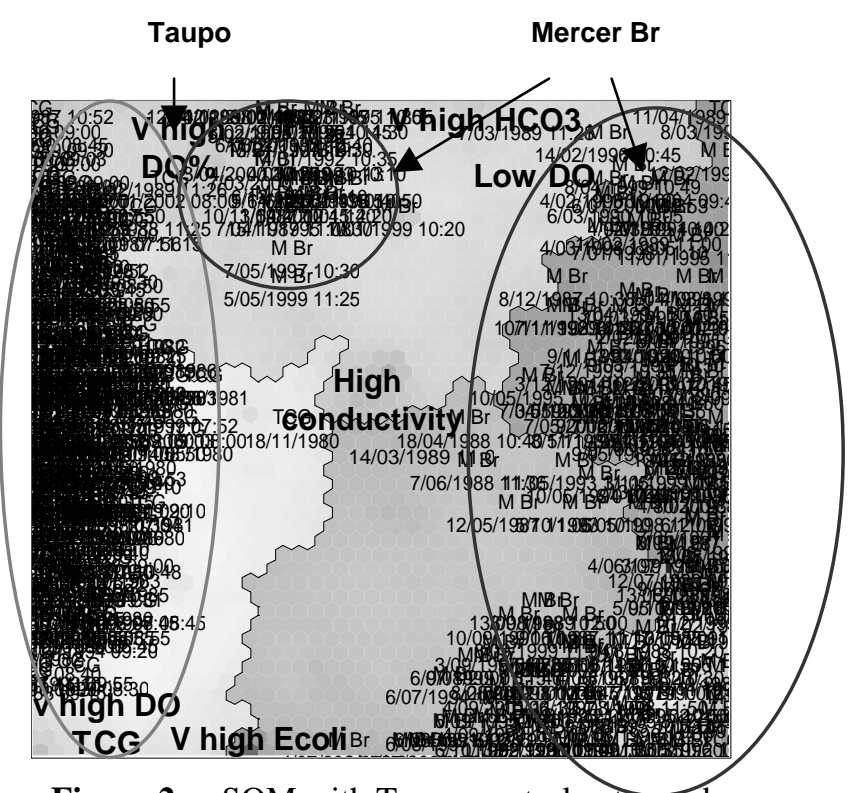

Figure 2 a: SOM with Taupo control gates and Mercer Branch data. 


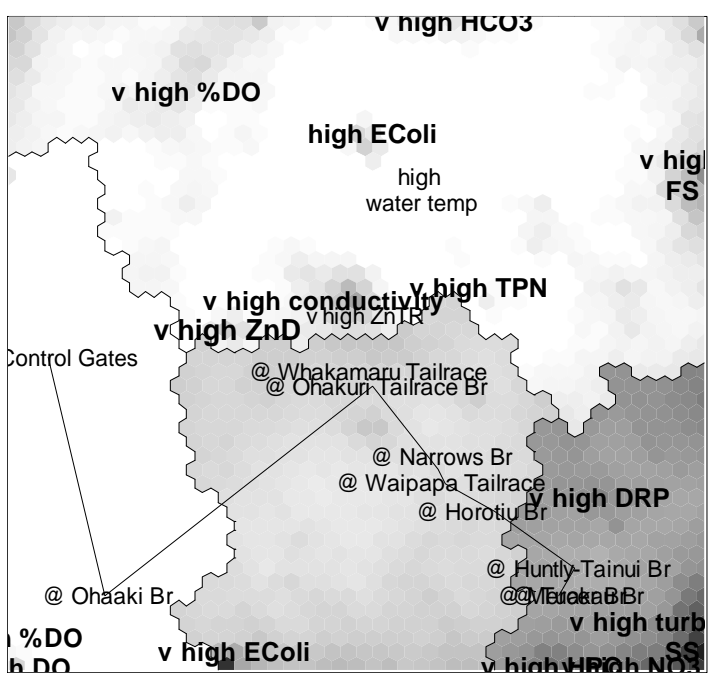

Figure 2b: Trajectory of the Wakato River on 4 June 2002 from different sites.

The following are the observations made from the animation of this trajectory:

(i) Dissolved oxygen (DO) decreased, with increased human use.

(ii) $\mathrm{NO}_{3}$ increased same as above.

(iii) Turbidity increased same as above.

(iv) Data points of 4 June 2002 are seen in the low temperature area, reflective of the wintry conditions.

\section{SOMS TO ANALYSE GLOBAL DATA}

A SOM (figure 3a) was created using the World Bank Report (2002) data on development related activities i.e. Gross Domestic Product (GDP), agriculture, industry, manufacturing, services and rural development with biodiversity indices of different countries to see the patterns in them.

\subsection{SOM results of global data}

The following are the interpretations arrived at from the SOM:

(i) Cluster 1, consisting of Brazil, Russian Federation and the United States in one node and with China and Indonesia in another, shows high and medium to high GDP, average annual percentage growth of agriculture, industry, manufacturing and services in 1980 to 1990 and 1990 to 2000 time periods. These counties also show medium to high numbers of mammals, birds, higher plants and high number of threatened species of these organisms. They also have large areas of protected land but when converted into percentage of total land area these countries have low percentages.
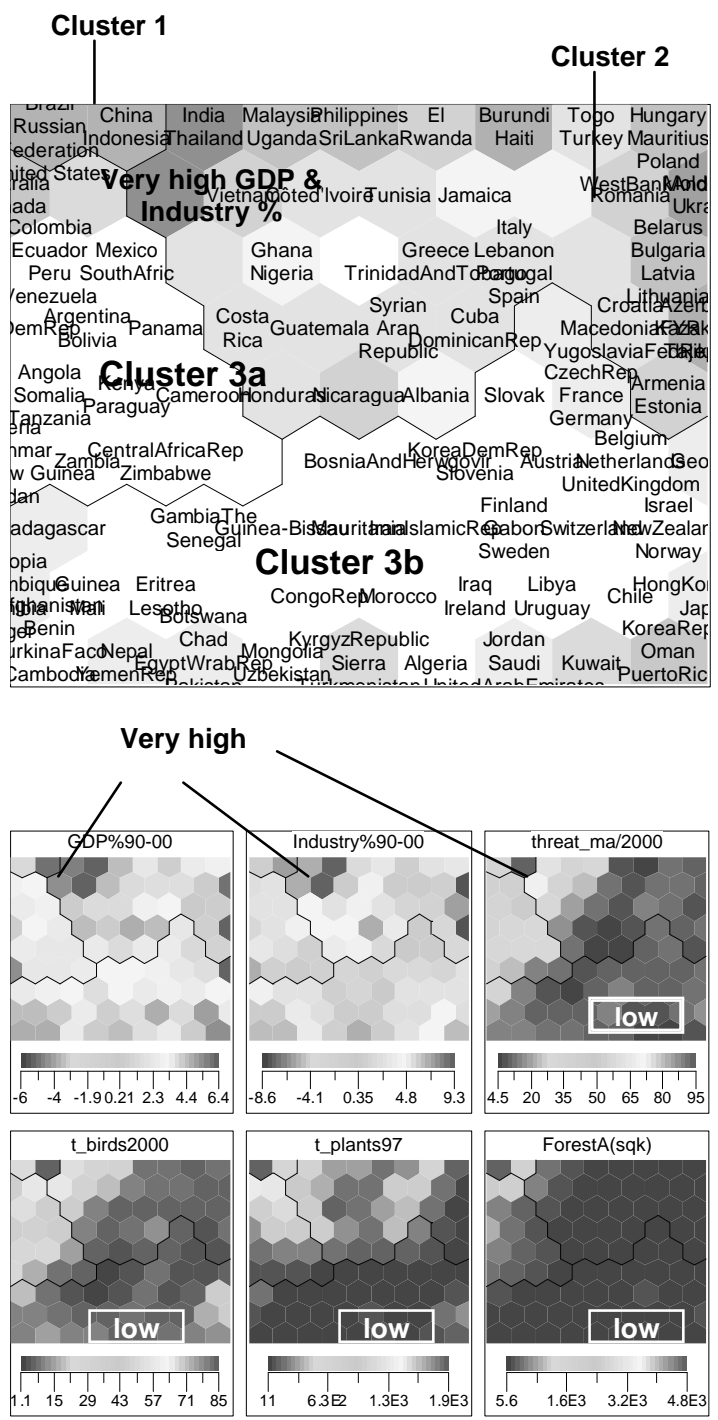

Figure 3a \& b: SOM created with development and biodiversity data of world countries. b: GDP, industry threaded mammals, birds, plants and forest $(90 / 00)$ components.

(ii) Within the cluster 1, China and Indonesia show the highest values of mammals and birds for 1996 as well as the highest rates of threatened species for both of them in the year 2000. These two counties also have enjoyed the highest GDP for both periods with high industry, manufacturing and percentage of average annual deforestation for (1990/2000); it could be interpreted that their GDP growth has come at the expense of biodiversity loss. The percentages of average annual deforestation of Brazil, Russian Federation and the United States do show higher GDP rates even though these countries do not match with those of China and Indonesia.

The total species numbers as well as the threatened species have a good correlation to forest area. This is considered to be confirming 
the WRI theories, to correlate forest area loss data to species diversity and loss.

The variables (i.e. agriculture, industry, manufacturing and services) attributing to the growth of development have good correlations within them during the time periods analysed (1980/1990 \& 1990/2000). The variables used are from the World Bank's pressure, state and response (PSR) indictor system report to analyse various ecosystems based on Hammond et al. (1995).

\section{SUMMARY}

The divide between the three major groups, scientists, stakeholders and the general public, who need to work together, is seen to be more widening as the demand on ecosystem use increases. Despite the efforts made by many state and international institutions to meet the need for integrated analysis of ecosystem and economic data, the issues remain the same. The examples of this paper illustrated how a refined approach of SOM analysis could provide a means to analyse ecological and economic data within an intergraded framework, understandable by a wider community.

\section{ACKNOWLEDGEMENTS}

The authors wish to thank the staff of Waikato Regional Council for permission to use their data. The literature review and the second example are excerpts of the main author's thesis submitted for a doctoral degree at the Auckland University of Technology, New Zealand.

\section{REFERENCES}

Bowler, P. J. (1992). The problem of perception. the fontana history of The environmental sciences. R. potter. London, Great Britain, HarperCollins Manufacturing, Glasgow: 634.

Buckeridge, J. S. (1994). "Introducing philosophy and ethics to the engineering curriculum." Transactions of the Institution of Professional Engineers New Zealand 21 Number 1/GEN.

Buckeridge, J. S. (2001). Ethics, Environment and Culture: Their significance within Engineering Education. International Ecological Engineering Conference CInternational Ecological Engineering Society (IEES) and New Zealand Engineers for Social Responsibility (ESR), Lincoln University.

Clark, J. S., S. R. Carpenter, N. Barber, S. Collins, A. Dobson, J. A. Foley, D. M. Lodge, M. Pascual, R. P. Jr, W. Pizer, C.
Pringle, W. V. Reid, K. A. Orse, O. Sala, W. H. Sschlesinger, D. h. Wall and D. Wear (2001). "Ecological Forecasts: An Emerging Imperative." The American Association for the Advancement of Science. Volume 293, Number 5530(Issue of 27 Jul 2001): 657-60.

Graedel, T. E., A. Alldredge, E. Barron, M. Davis, C. Field, B. Fischhoff, R. Frosch, S. Gorelick, E. A. Holland, D. Krewski, R. J. Naiman, E. Ostrom, M. Rosenzweig, V. W. Ruttan, E. K. Silbergeld, E. Stolper and B. L. T. II (2001). Grand Challenges in Environmental Sciences. Washington, National Academy Press: 96.

Gustavsson, L. (2001). On Ecological Engineering and Sustainable Development in a Swedish Context. International Ecological Engineering Society (IEEE) and Engineers for Social Responsibility (ESR), Lincoln University, New Zealand.

Hammond, A., A. Adriaanse, E. Rodenburg, D. Bryant and R. Woodward (1995). ENVIRONMETAL INDICATORS - A systematic approach to measuring and reporting on environmental policy performance in the context of sustainable development, World Resources Institute.

Harris, G. (2002). Integrated assessment and modelling: an essential way of doing science. Science Direct, Environmental Modelling and Software, Volume 17: 201-207.

Mann, K. H. (1982). Models and Management. Ecology of Coastal Waters - A Systems Approach, Oxford : Blackwell Scientific Studies in Ecology. Volume 8: 269.

Reid, W. V. (2000). The Delicate Balance: Environment, Economics, Development Ecosystem Data to Guide Hard Choices. ISSUES IN SCIENCE AND TECHNOLOGY.

Shanmuganathan, S., P. Sallis and J. S. Buckeridge (2001). Self-organising maps to study the effects of urbanisation at Long Bay in New Zealand,. Proceedings of the Fifth Biannual Conference on Artificial Neural Networks and Expert Systems, Dunedin , New Zealand.

Vant, B. (1999). Analysing and Reporting SoE Monitoring Results for Rivers in the Waikato Region. Proceedings of the Environmental Indicators Symposium, University of Otago.

Waikato Regional Council (2003). Waikato River: A Water Quality Monitoring Map. Waikato, Waikato Regional Council.

World Bank Report (2002). 4.1 Growth of output. 